\title{
Daviel et Genève
}

Conférence présentée le 29 octobre 1976 au Colloque «Genève et la Médecine», Genève

Par Albert Franceschetti

\section{Introduction}

Jacques Daviel, éminent chirurgien oculiste, vint à Genève en 1762 dans le seul but de s'y faire soigner par Théodore Tronchin (1709-1781), médecin alors à la mode et partisan de «la Nature, la grande guérisseuse». Daviel n'y passera que quinze jours de son vivant, mais l'importance de la révolution chirurgicale qu'il introduisit, le retentissement de son œuvre et le fait qu'il soit enterré en sol genevois, mérite que l'on s'attarde sur l'histoire de ce médecin extraordinaire.

\section{Histoire}

Jacques Daviel nacquit le 11 août 1696 à la Barre près de Rouen, où son père était tabellion royal. Adolescent, il entre en apprentissage chez son oncle, chirurgien à Rouen, et à l'âge de 17 ans nous le retrouvons déjà chirurgien aide-major aux armées. Il continue ensuite ses études à l'Hôtel-Dieu de Paris.

Lors de la peste de 1720 , il se joint en tant que volontaire à l'équipe parisienne qui se rend en Provence pour soigner les malades et il continuera de se consacrer à cette tâche jusqu'à la fin de l'épidémie, en 1722. Il se fixe alors à Marseille où, tout en poursuivant son activité professionnelle, il ouvre un cours d'Anatomie et Chirurgie. Son intérêt pour la chirurgie oculaire l'amène, à partir de 1728, à se consacrer entièrement à cette branche. Sa réputation grandissante dépasse peu à peu les frontières, si bien qu'en 1736 il est appelé au Portugal et en Italie. Enfin le 8 avril 1745 se produisit l'évènement décisif de sa vie. Nous en trouvons le récit dans les Mémoires de l'Académie de Chirurgie de Paris:

Un hermite d'Aiguilles en Provence ayant été opéré sans succès d'une Cataracte à l'œil droit, vint me trouver à Marseille pour me prier de l'opérer du gauche. Je ne fus pas plus heureux que celui qui l'avoit opéré d'abord. M'étant servi de l'aiguille ordinaire tranchante sur les côtés, non-seulement je ne pus abattre la Cataracte, mais il arriva encore que plusieurs portions du cristallin brisé passèrent dans la chambre antérieure que je vis se remplir de sang pendant l'opération, de façon que mon aiguille ne paroissant plus, je fus obligé de la retirer sans pouvoir achever l'opération. Cet accident me détermina, à l'exemple 


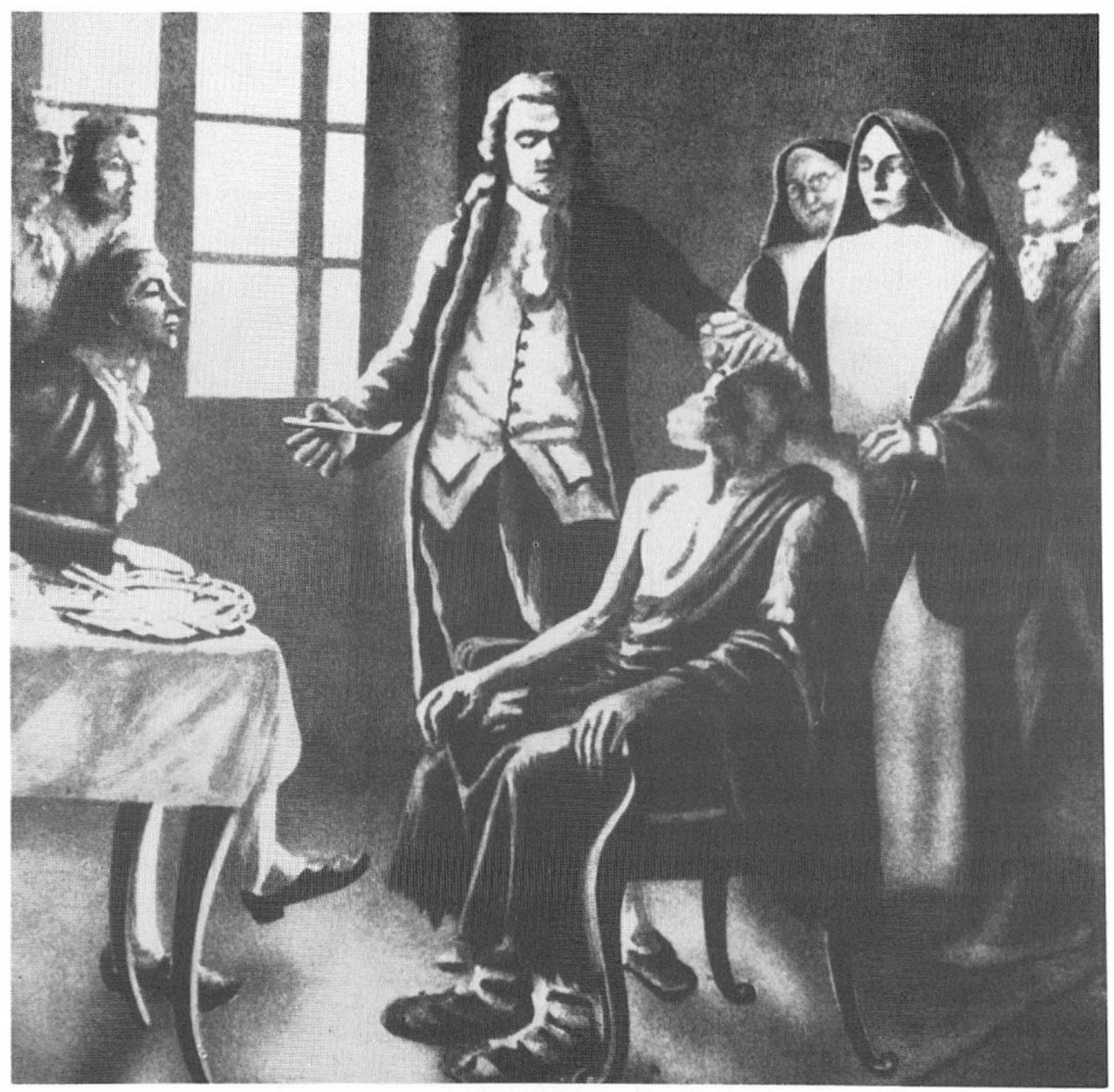

Fig. 1. Tableau de E. Mein (1925)

de M. Petit, à ouvrir la cornée transparente pour évacuer le sang et les fragments de la Cataracte qui avoient passés dans la chambre antérieure, ce que je fis en portant une aiguille demi-courbe dans cette chambre, j'agrandis la première ouverture de la cornée avec des petits ciseaux courbes, et par ce moyen tout ce qui étoit dans la chambre antérieure s'évacua, la Prunelle parut nette, et le malade distingua aussi-tôt les objets qui lui furent présentés ;...

En 1925, le peintre E. Mein peignit cette scène en y faisant figurer quatre oculistes contemporains (fig. 1, de gauche à droite, le Professeur Aubaret, le Docteur D. Hermann de Genève, le Docteur Jean Sédan et le Docteur Solari). 
Le $1^{\text {er }}$ janvier 1749, Daviel est nommé oculiste du roi Louis XIV, ce qui consacre sa célébrité. Preuve en soit l'ode parue au Mercure de France en juillet 1752, embellie d'un dessin allégorique, qui le montre entre son génie et la Médecine (fig. 2).

Entre 1750 et 1760, Daviel est appelé auprès de plusieurs cours européennes et, au cours de ces voyages, il pratique de nombreuses interventions.

Cependant depuis la fin de 1761, Daviel éprouve - «une sorte d'embarras de la langue, qui gênoît un peu la parole, laquelle gêne il attribuait à la viscosité de la salive, quoiqu'il ait employé bien des remèdes, peut-être assez mal à propos, méprisant en cela les conseils des médecins. Le mal a fait des progrès si rapides que déjà au mois de mars de cette année (1762), il étoit tombé en complète aphonie.» Telles sont les paroles de Monsieur d'Apples, médecin de Lausanne, qui s'occupa également de soigner Daviel.

En 1762, il doit faire lire par un collègue le mémoire qu'il a préparé pour l'Académie de Chirurgie de Paris. Durant l'été, il opère en province et se rend aux Eaux de Bourbon l'Archambault, lesquelles ne lui sont d'ailleurs d'aucun secours.

Au début de septembre, se trouvant à Lyon, il prend la résolution d'aller consulter Tronchin à Genève. Il en fait l'annonce à Albert de Haller, dans une lettre datée du 12 septembre.

Au milieu du mois, il arrive à Genève. Une chaise de poste venant de Lyon s'arrête place Bel-Air et - nous raconte l'historien Vetter - «l'animation gagne les nombreux aristocrates et les grandes dames de la société française accoudés aux fenêtres de l'Hôtel des Balances, lorsqu'ils reconnaissent l'oculiste du Roi de France».

«Pour mieux revivre la scène, il nous faut regarder la vue de la place Bel-Air et de l'Isle, peinte et gravée par Geissler, quelques décennies plus tard. C'est dans cette hostellerie, facilement reconnaissable à l'enseigne rectangulaire, que Jacques Daviel a passé ses derniers jours. L'aubergiste s'empresse pour accueillir le célèbre voyageur qui s'exprime par gestes» (fig. 3 a).

Daviel consulte donc Tronchin, mais le traitement tardif que lui donne ce médecin ne le soulage aucunement. Il décède le 30 septembre 1762 à 6 heures du matin, probablement des suites d'un cancer du larynx. Tronchin, ayant pratiqué l'autopsie, ne trouve apparemment qu'un amincissement et une anémie des muscles du larynx et du pharynx.

Selon ses dernières volontés et grâce à l'intervention du résident de France, Monsieur de Montperaux, Daviel est enterré au cimetière du Grand Saconnex, alors terre catholique et française. 


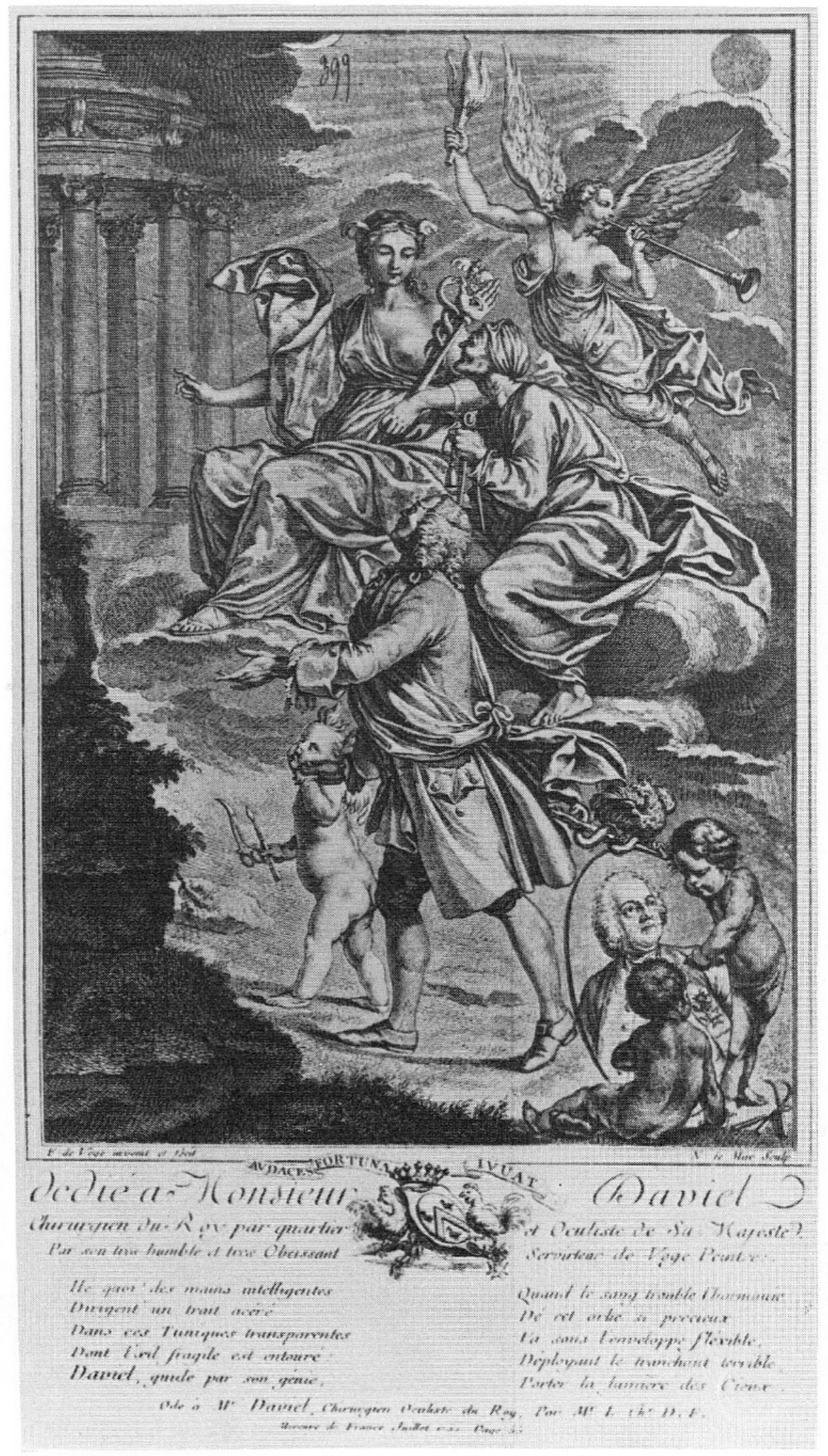

Fig. 2. Allégorie et ode parues au Mercure de France (1752) 

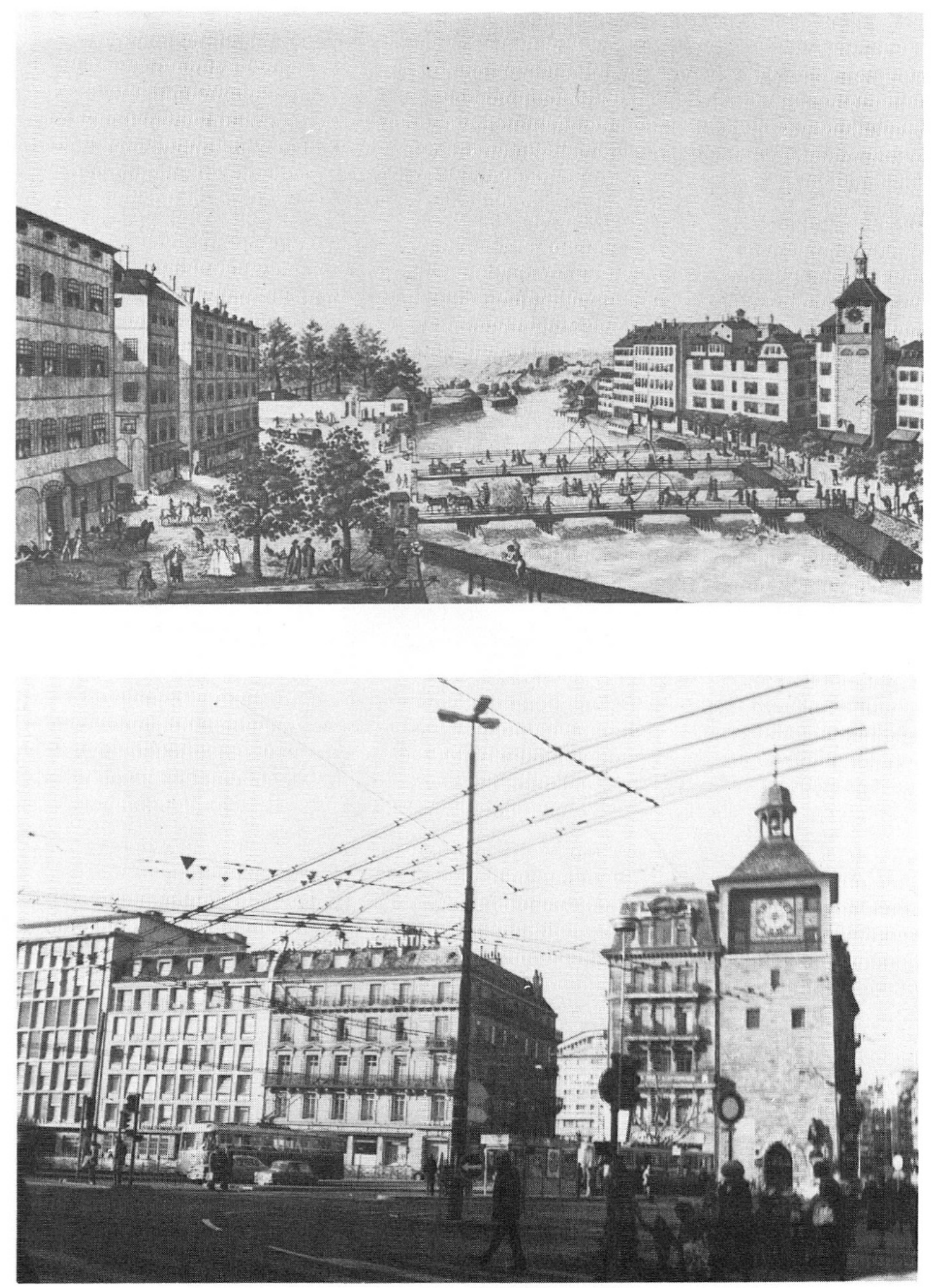

Fig. 3. Vues de la Place Bel-Air, a) par Geissler au XVIII ${ }^{\mathrm{e}}$, b) en 1976 
Analyse

Si nous voulons comprendre la renommée de Daviel parmi ses contemporains, il nous faut considérer les résultats de ses opérations de cataracte. Jean Sédan, autre illustre ophtalmologue de Marseille, mais celui-ci notre contemporain, cite 182 résultats parfaits sur 206; Hirschberg en cite 384 sur 434, performances à peine croyables pour l'ère pré-aseptique.

Il faut cependant attendre jusqu'en 1885 pour que les ophtalmologues fassent un geste de reconnaissance vis-à-vis de Daviel. C'est tout à l'honneur de la faculté de Médecine de Genève, dont nous fêtons le centenaire, que ce soit un oculiste genevois à en avoir pris l'initiative. Le 8 octobre 1885, Monsieur Haltenhoff, futur premier Professeur d'Ophtalmologie à la Faculté de Médecine, inaugure un monument à la mémoire de Jacques Daviel au cimetière du Grand Saconnex. Le texte, gravé sur la pierre, porte les mots suivants (fig. 4) : «Jacques Daviel, né à la Barre en 1696, mort à Genève en 1762, chirurgien et oculiste, qui le premier, guérit la cataracte par extraction, repose dans ce cimetière - érigé par les oculistes suisses, 1885 ». Relevons que la date de naissance est inexacte car Daviel naquit en 1693.

Ce monument, érigé sur proposition de Haltenhoff, fut financé par une souscription nationale et internationale, patronnée par Horner (Zurich), Dufour (Lausanne) et le même Haltenhoff (Genève). La stèle est due à l'architecte Emile Reverdin et le médaillon au sculpteur Hugues Bovy (fig. 4).

Le texte du discours prononcé par Haltenhoff, lors de l'inauguration du monument, place admirablement Daviel et illustre l'importance de sa découverte pour les ophtalmologues. Ce texte est extrait de la Revue médicale de la Suisse romande.

«Messieurs,

Avant de soulever les voiles qui couvrent cette pierre, laissez-moi vous rappeler en peu de mots les droits que s'acquit Jacques Daviel à l'admiration et à la reconnaissance de la postérité. Vers le milieu du XVII ${ }^{\text {ème }}$ siècle, l'art de guérir était à peine émancipé des entraves de la tradition scolastique, toute puissante au Moyen-Age. Le mouvement progressif, qui devait en faire une branche des sciences naturelles, avait commencé, il est vrai, grâce aux travaux des grands anatomistes. Mais l'oculistique n'avait guère profité de ces progrès. Elle restait en grande partie entre les mains de charlatans ignorants. L'opération de la cataracte, la plus importante de la chirurgie oculaire, était couramment pratiquée sur les places publiques par des oculistes ambulants. Elle consistait à abaisser, dans le fond de l'œil, au moyen d'une aiguille, le cristallin devenu opaque, afin d'en débarrasser la pupille. Cette opération avait en général un succès immédiat et le malade recouvrait la vue, mais trop souvent il sur- 


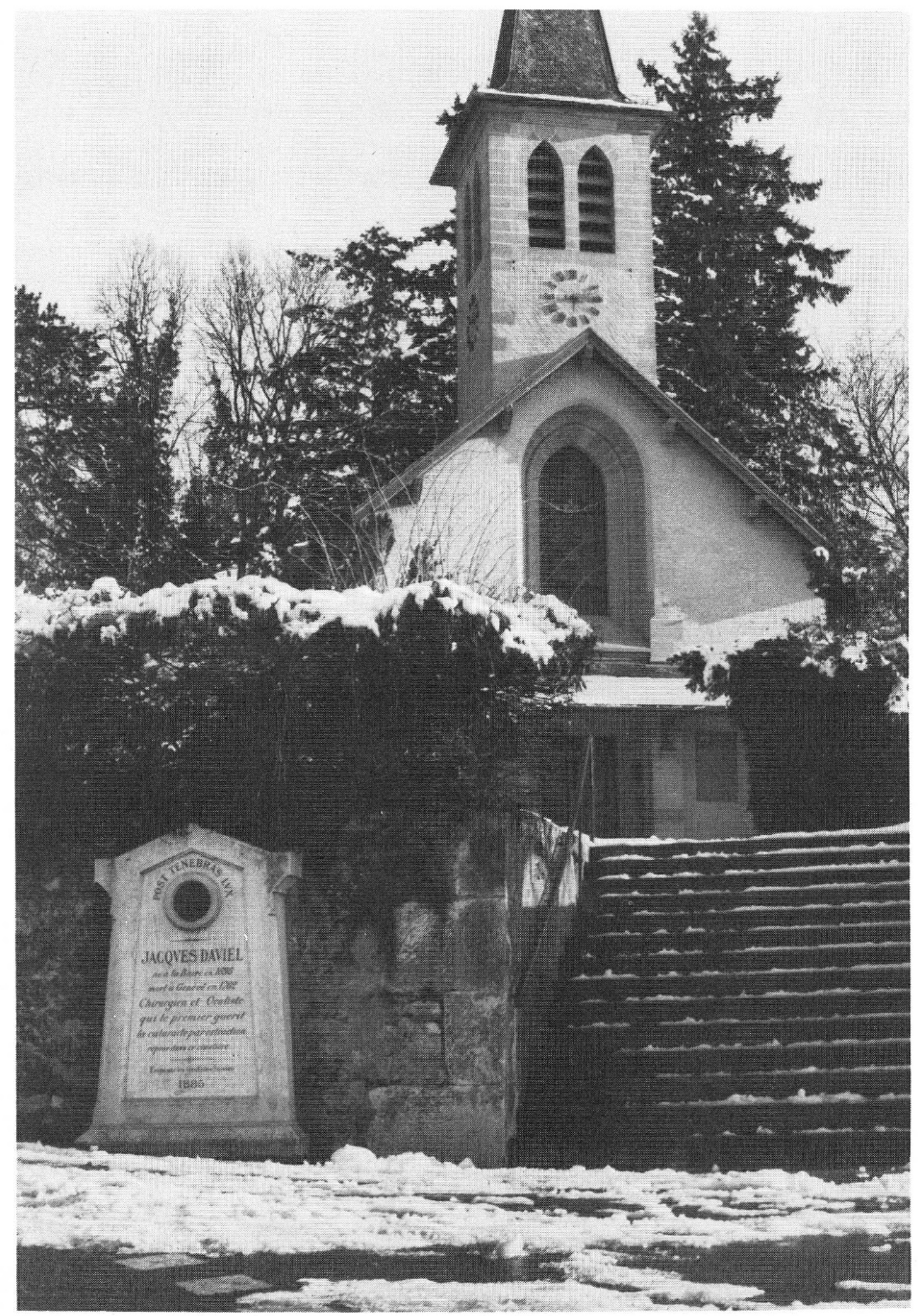

Fig. 4. Monument érigé par les ophtalmologues suisses (1885) 
venait bientôt après une lente inflammation du globe oculaire et dans la majorité des cas la vue se perdait sans espoir de retour, au prix de longues souffrances. Parfois même, l'autre œil était aussi détruit par une inflammation sympathique. Une longue série de siècles avait consacré les principes et le manuel de cette opération dont, en somme, aucun perfectionnement notable n'avait modifié les résultats. Jacques Daviel s'éleva le premier contre cette pratique séculaire de l'abaissement. Il déclara hardiment que l'on devait extraire de l'aeil la cataracte, c'est-à-dire le cristallin malade...»

Plus loin, Haltenhoff ajoute: «Ce n'est guère que vers le milieu du XIX ème siècle que l'extraction fut adoptée universellement comme seule opération normale de la cataracte. Le procédé opératoire et les instruments ont subi depuis Daviel bien des modifications, mais le principe est toujours le même...» et il conclut : «si chaque année, dans tous les pays du monde, des milliers d'aveugles recouvrent, pour le reste de leurs jours, le sens précieux de la vue, c'est à Daviel qu'on le doit».

L'initiative de Haltenhoff éveilla l'attention de quelques personnalités habitant la ville de Bernay, chef-lieu de l'arrondissement où naquit Daviel. Le 22 novembre 1885, un certain Docteur Gauron prononça l'éloge de Daviel devant la «Section de Bernay de la Société libre de l'Eure». A la suite de cela, la société nomma une commission ayant pour mandat «d'aller faire les démarches nécessaires auprès des sommités médicales de Paris». Le comité pour l'érection en France d'un monument à la mémoire de Jacques Daviel fut présidé par Panas, Professeur à la Faculté de Médecine de Paris, et comprenait entre autre Haltenhoff.

C'est en juillet 1891 qu'a lieu l'inauguration du monument de Daviel à Bernay et le Docteur Chavernac d'Aix prononce un discours, débité «avec une chaleur toute méridionale et une éloquence entrainante », dont j'extraîs le paragraphe concernant la Suisse :

«Notre illustre confrère alla mourir en Suisse. Remercions le savant Dr. Haltenhoff de lui avoir fait élever un monument qui, en perpétuant le souvenir du grand oculiste français, nous rappellera toujours l'action généreuse de nos confrères helvètes et cette vaillante nation qui a sculpté sur sa frontière la légende républicaine : «Intelligence et Liberté». (Tonnerre d'applaudissements.)»

Pour le $200^{\mathrm{e}}$ anniversaire de la mort de Daviel en 1962, René Onfray publie un esquisse cinématographique, où il nous fait revivre les scènes marquantes de la vie de Daviel (fig. 5).

En 1963, à l'occasion du $70^{\mathrm{e}}$ anniversaire de la Société Française d'Ophtalmologie, Monsieur Vetter, de la Société Française d'Histoire de la Médecine, met 


\section{CONFERENCES LYONNAISES D'OPHTALMOLOGIE

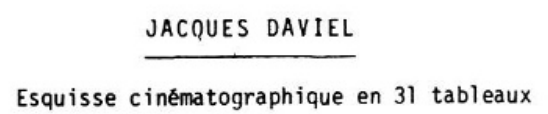

R. ONFRAY

xivil

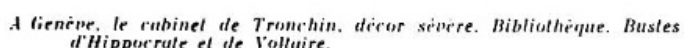

IX VAllat (it introduit Damiel et M. d'Apples)

Mensicur le Professeur allend la visile de ece Yessieurs, mais il a ele appele ehez .I. de Vollaire. il ne saurait tarder i venir.

Isseyez-vous, mon cher Monsicur Daviel. DAVItI. (trune mi.r rumque a peine perceptible)

Merci, Monsieur, je crains bien que celte consultation ne soit inutile.

D'APPLES

Mais nen, cher Monsicur. 'Tronchin est tries habile, il aur des remedes que ne conmaissent pas nos confreres des caux du Bourbon. II. de Vollaire, qui est si fin, ne jure que par lui, e'est une bonne référence.

Davitl (à poir éleinte)

Ah ! ces caux de Bourben m'ont élé nuisibles, el ce vovagé par Lyon m'a bien fatigué. Quelle misere de ne pouvoir parler!

Entre Tronchin qui s'incline.

TRONCHIX Excusez-moi, Mlessicurs. d'ètre un peu en retard. M. de
Voltaire m'avait fait appeler el vous savez qu'il est peu patient D'APPLES

Cher Maitre, comme je vous l'ai mandé de Lausanne, je vous amene M. Davicl. Il a doére encore hout recemment avec succès M. de Forel et celui-ci l'a persuadé de vous venir con-
sulter pour cette aphonie qui l'inquiète, et peine tous ses opé-

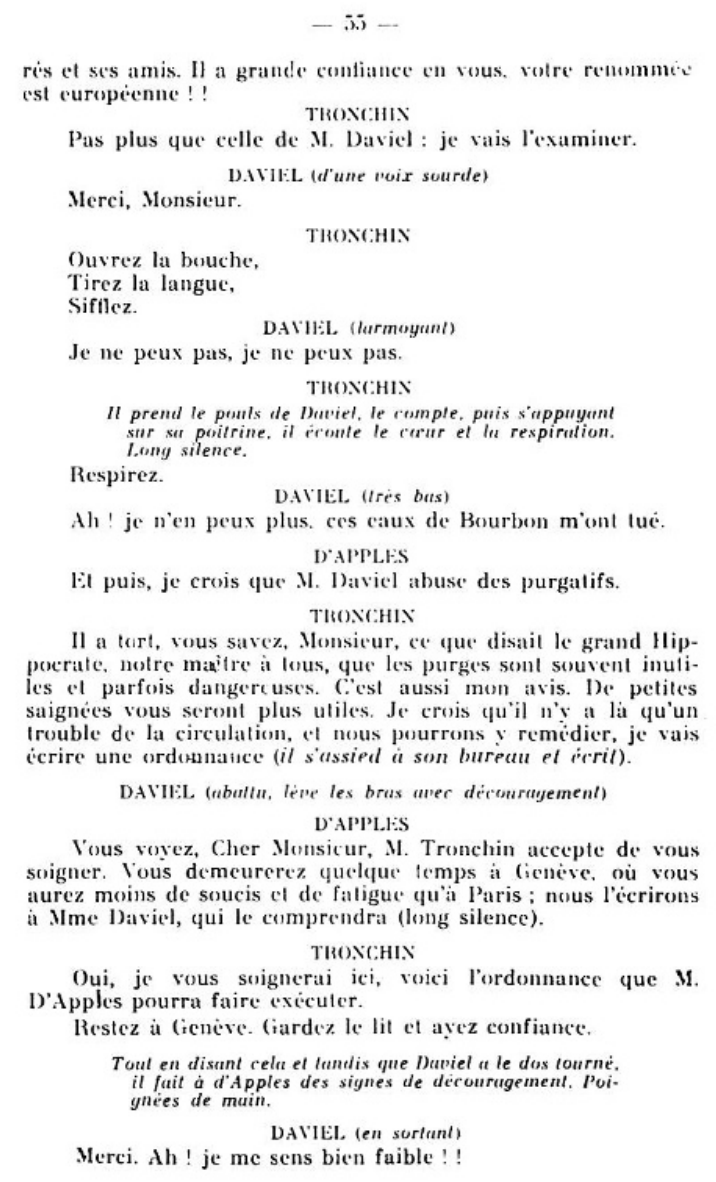

Fig. 5. Esquisse cinématographique de R. Onfray (1962)

sur pied une exposition et un catalogue détaillé des documents se rapportant à Jacques Daviel, y compris ceux concernant la période genevoise de celui-ci. Mentionnons en passant que cette même année, le rapport annuel devant la Société Française était présenté par deux genevois, le $3^{\text {e }}$ Professeur d'Ophtalmologie, Adolphe Franceschetti, et le futur $4^{\mathrm{e}}$ Professeur, Jean Babel.

Ajoutons encore que Daviel, genevois de par sa mort, figure sur un timbre des postes françaises.

Du catalogue de Vetter, je vous ai montré la vue de la place où se trouvait l'élégant Hôtel des Balances. Par comparaison, la Place Bel-Air d'aujourd'hui montre le changement profond qu'a subit Genève (fig. $3 \mathrm{~b}$ ). Le rayonnement de la ville ainsi que de sa Faculté de Médecine n'a certes pas diminué; bien au contraire, il n'a cessé de croître au cours de ces deux cents ans. 


\section{Bibliographie}

Anonyme, Genf. Monument Daviel. Corresp. Blatt Schw. Ärzte 23 (1885), p. 582-583.

Anonyme, Inauguration du monument de Daviel. Revue méd. Suisse romande 5 (1885), p. 617-621.

Chavernac, Daviel en Provence. J. et A. Lefèvre, Bernay 1891, 8 p.

Daviel, J., Sur une nouvelle méthode de guérir la cataracte par l'extraction du cristallin. Mém. Acad. royale Chir. 2 (1753), p. 337-354.

Delacroix, H., Jacques Daviel à Reims. G. Masson, Paris 1890, 90 p.

Duke-Elder, S. (Sir), Surgical treatment of cataract. History. In: Duke-Elder, S., Diseases of the lens and vitreous; Glaucoma and hypotony. System of Ophthalmology, vol. XI, p. 248-263, H. Kimpton, London 1969.

Filippi-Gabardi, E., Jacques Daviel e l'accademia delle scienze dell'Istituto di Bologna. Giorn. Ital. Oftal. 10 (1957), p. 81-101.

Gautier, L., La médecine à Genève jusqu'à la fin du dix-huitième siècle. Georg, Genève 1906, $696 \mathrm{p}$.

Haltenhoff, G., Courte notice historique sur Jacques Daviel. Rev. méd. Suisse romande 4 (1884), p. 599-600.

Hirschberg, J., Jacques Daviel. In: Graefe/Saemisch, Handbuch der Augenheilkunde, II éd., vol. XIII, p. 470-546, Engelmann, Leipzig 1908.

Kronfeld, P., Jacques Daviel: The inventor of cataract extraction. Survey Ophthal. 14. (1969-70), p. 118-122.

Onfray, R., Jacques Daviel. Esquisse cinématographique. Conf. lyonnaise ophtalm. (Lab. H. Faure), No. 63 (196/2), p. 1-61.

Sédan, J., La révolution opératoire de JJacques Daviel. Clin. ophtalm. (Lab. Martinet) 1 (1963), p. 3-14.

Simon de Guilleuma, J. M., Jacobo Daviel. Oculista del Rey Luis XV, Su instrumental, biografia y viajes. Lab. Norte España, 1943, 39 p.

Vetter, Th., Rencontres avec Jacques Daviel. Exposition présentée à l'occasion du $70^{\mathrm{e}}$ Congrès de la Société Française. Centre Marcelin-Berthelot, Paris 1963, 57 p.

Vetter, Th., Jacques Daviel en France et en Europe. Clin. ophtalm. (Lab. Martinet) 1 (1963), p. 17-27.

\section{Summary}

Jacques Daviel came to Geneva in 1762 to consult the famous Dr. Tronchin but died soon thereafter. He was buried in le Grand Saconnex near Geneva. In 1885, a monument was erected to the memory of this famous ophthalmologist, who had introduced the extraction of the cataract.

Dr. med. Albert Franceschetti

4 , rue Bellot

1208 Genève 